\title{
Correction to: Hierarchy of hair loss stigma: media portrayals of cancer, alopecia areata, and ringworm in Israeli Newspapers
}

Daphna Yeshua-Katz ${ }^{1 *}$, Shifra Shvarts ${ }^{2}$ and Dorit Segal-Engelchin ${ }^{3}$

\section{Correction to: Isr J Health Policy Res \\ https://doi.org/10.1186/s13584-019-0338-0}

The original publication of this article [1] contained an incorrect title. The correct and incorrect information are shown below:

Incorrect: Hierarchy of hair loss stigma: media portrayals of cancer, alopecia areata, and cancer in Israeli newspapers

Correct: Hierarchy of hair loss stigma: media portrayals of cancer, alopecia areata, and ringworm in Israeli Newspapers

The original publication has been updated.

\section{Author details}

'Department of Communication Studies, Ben-Gurion University of the Negev, POB 653, Beer-Sheva, Israel. ${ }^{2}$ Moshe Prywes Center for Medical Education, Faculty of Health Sciences, Ben-Gurion University of the Negev, Beer-Sheva, Israel. ${ }^{3}$ Spitzer Department of Social Work, Ben-Gurion University of the

Negev, Beer-Sheva, Israel.

Published online: 14 October 2019

\section{Reference}

1. Yeshua-Katz D, Shvarts S, Segal-Engelchin D. Hierarchy of hair loss stigma:

media portrayals of cancer, alopecia areata, and ringworm in Israeli newspapers. Isr J Health Policy Res. 2019;8:68 https://doi.org/10.1186/ s13584-019-0338-0.

\footnotetext{
* Correspondence: yeshuad@bgu.ac.il

${ }^{1}$ Department of Communication Studies, Ben-Gurion University of the Negev, POB 653, Beer-Sheva, Israel

Full list of author information is available at the end of the article
} 